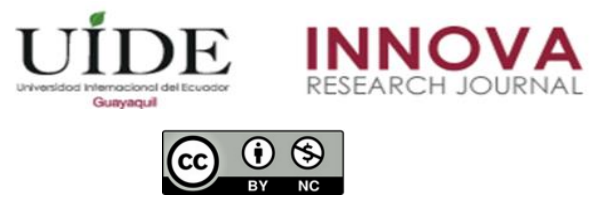

INNOVA Research Journal, ISSN 2477-9024

(Enero-Abril 2020). Vol. 5, No.1 pp. 94-110

DOI: https://doi.org/10.33890/innova.v5.n1.2020.1159

URL: http://revistas.uide.edu.ec/index.php/innova/index

Correo: innova@uide.edu.ec

\title{
Influencers ecuatorianos en el proceso de posicionamiento de su marca personal
}

\section{Ecuadorian influencers in the process of positioning your personal brand}

Geovanny Fernando Zhapán Revilla

Universidad Técnica de Machala, Ecuador

Autor por correspondencia: gzhapan_est@utmachala.edu.ec

Fecha de recepción: 16 de agosto del 2019 - Fecha de aceptación: 31 de octubre del 2019

\section{Resumen}

El presente trabajo usó un método cualitativo para analizar el patrón de comportamiento de los influencers ecuatorianos con el fin de entender su posicionamiento de marca personal en el país, lo cual ha sido considerado como un caso de estudio. El objetivo que se planteó fue identificar cómo los influencers ecuatorianos han usado las redes sociales como medio de comunicación para posicionar su marca personal. Los resultados indican que la mayoría de influencers que se tomaron en cuenta para este estudio usan el entretenimiento (cómico) como método de captar la atención de sus seguidores y aumentar su alcance local a nivel nacional. Se concluye con una perspectiva panorámica de la herramienta que utilizan los influencers para colocarse en un sitio donde su marca personal pueda ser vista y así mismo su patrón de comportamiento, se proporciona más información de esto, en el desarrollo del artículo detallado con una clasificación del objeto de estudio y los resultados encontrados.

Palabras claves: influencers; redes sociales; marca personal; contenido no comercial; patrones de comportamiento

\begin{abstract}
The present work used a qualitative method to analyze the behavior pattern of Ecuadorian influencers in order to understand their personal brand positioning in the country, which has been considered as a case study. The objective was to identify how Ecuadorian influencers have used social networks as a means of communication to position their personal brand. The results indicate that the majority of influencers that were taken into account for this study use (comic) entertainment as a method of capturing the attention of their followers and increasing their local reach nationwide. It concludes with a panoramic perspective of the tool that influencers use to place themselves in a place where their personal brand can be seen and also their behavior pattern, more information about this is provided, in the development of the detailed article with a classification of object of study and the results found.
\end{abstract}

Key words: influencers; social networks; personal brand; non-commercial content; behavior patterns 


\section{Introducción}

En la actualidad se ha podido observar cómo ha evolucionado la publicidad por lo que las empresas buscan formas más efectivas para ganar reconocimiento en el mercado. Un elemento muy importante es el uso de los influencers, los mismos que han logrado posicionarse a través de su marca personal. Estos prescriptores día a día están al pendiente de las nuevas tendencias siendo esto muy atractivo para los miles de seguidores de sus plataformas.

La publicidad día a día va mejorando y cambiando aspectos fundamentales para las personas y organizaciones, cada vez los procesos promocionales son menos complejos y más adaptados a las tendencias sin dejar de lado el informar y persuadir a un segmento previamente definido.

Las empresas están adaptándose a estos nuevos procesos aplicando estrategias que le permitan convencer a su audiencia haciéndolo de manera didáctica y entretenida, para esto estudian el mercado y conocen previamente lo que desea y espera el cliente (Moreno y Calderón, 2017).

Es así como se ha podido observar que en las empresas están cambiando los medios de difusión de la publicidad, puesto que era muy notorio ver a las empresas pautar en televisión, radio, periódico, páginas amarillas; este tipo de actividades no son realizadas con frecuencia en esta época, porque el auge del internet ha logrado que existan cambios relativos en los medios de comunicación y por ende medios publicitarios, en la actualidad es muy común ver a las empresas crear páginas en redes sociales, tener dominios de página web (Salas, 2009).

Ante esto se plantea como ejemplo el caso de España, encontramos como referencia Arce y Cebollada (2013) en el 2007 el 53\% de una población de 14 años tenía acceso a internet, un $39,8 \%$ había concretado compras online durante ese periodo. El monto de gasto por comprador fue de 595 euros. Durante el 2008, en las ventas online se registraron 6.400 millones de euros, un $34 \%$ más que en el año 2007. Durante este periodo, se registró un alza en la compra de boletos de transporte, productos de consumo masivo y entre otras cosas.

Los enfoques del marketing y publicidad han logrado que en las empresas exista mayor preocupación por el consumidor, importa mucho lo que ellos piensan y cómo actúan; por este motivo es que el marketing se relaciona con otras disciplinas como la economía, la psicología, la biología, entre otras; las mismas que le permiten llevar un conocimiento en función del comportamiento del consumidor, sus gustos y necesidades.

Y es que precisamente las redes sociales se han convertido en una herramienta clave para que las empresas hagan publicidad de sus productos o servicios, lo fundamental de este tipo de publicidad es el largo alcance que tienen, es decir, el anuncio publicitario llega de forma sincronizada al segmento específico de la empresa, impactando al usuario y generando deseos de compra.

Con la implementación de la publicidad en redes sociales surgen los conocidos influencers, siendo estas personas líderes de opinión porque son reconocidas en el medio 
televisivo que han logrado posicionar su marca personal a través del uso de las redes sociales, existen diversas temáticas en las que se desempeñan, emitiendo contenido informativo, humorístico, deporte, moda, belleza, salud, cocina, entre otros; siendo estos los motivos que llevan a muchos seguidores desear ser como ellos.

Es esto lo que aprovechan muchas marcas, evalúan a los influencers más queridos y los contratan para que hagan publicidad de sus productos/servicios en sus redes sociales, para que todos sus seguidores se sientan atraídos e identificados, creando además la necesidad de comprar para poder utilizar los mismos productos que su prescriptor favorito. (Taramona, 2018)

Involucrar al consumidor es la temática de estudio que se ha tomado desde la teoría del Comportamiento del Consumidor, en la que se encuentra encabezando la representación del involucramiento como dependiente de indicadores psico-emocionales.

La organización del relacionamiento de mercado propone que, de los elementos para el correcto funcionamiento el compromiso es uno de los más necesarios y que está presente en relaciones de intercambio, entendiéndolo bajo el concepto de Involucramiento o Implicancia (Duque y Ramírez, 2014).

Por este motivo el objetivo de la presente investigación está orientado a identificar cómo los influencers ecuatorianos han usado las redes sociales como medio de comunicación para posicionar su marca personal. Todo esto será para determinar el comportamiento de los prescriptores y el motivo que hace que muchas empresas quieran publicitar sus marcas con ellos.

El estudio de los influencers y su marca personal es importante porque se trata de un tema de actualidad, siendo un fenómeno que en matices académicas ayudarían a obtener conocimientos en relación a su comportamiento, evolución, adaptación en el mercado y al producto que ofrecen a sus seguidores los cuales están al pendiente de cada uno de sus actividades diarias.

Se ha podido observar que la relación que tienen los influencers con su audiencia bordea lo íntimo, porque comparten cada una de sus actividades, los hacen partícipes de sus triunfos, cuentan a su audiencia sus experiencias y estilo de vida, motivo por el que muchos suscriptores se identifican con estos personajes, queriendo adoptar las tendencias que en ellos observan y deseando estar a la moda incluyéndose en su estilo de vida.

La presente investigación tiene un enfoque cualitativo, en donde se va a determinar a través de una guía de observación On-line los factores más relevantes que permiten el posicionamiento de la marca personal en los usuarios/suscriptores/seguidores.

Se analizarán aspectos como número de seguidores, contenido que presentan en sus redes sociales, segmento al que se dirige el influencer, frecuencia de publicaciones, interacción con los seguidores, marcas que contratan servicios de los prescriptores.

Y así llegamos al tema de que la profesionalización de los Influencers y su marca personal no son muy fundamentadas en investigaciones académicas, pero no por eso es menos 
importante, su alcance es muy extenso en muchos de los casos, como el claro ejemplo del sueco PewDiePie que, tiene 60 millones de subscriptores y sus ingresos anuales bordean los 12 millones de dólares.

Los influencers son aquellos individuos que, por su carisma son capaces de comunicar y conectar de una forma casi íntima con el público, su fama en la internet los ha hecho generar un ejército de seguidores en las redes sociales (Villena, 2018).

Por otro lado, no se trata solo de su alcance numérico lo que refleja su importancia de estudio: sino también que denota a los Influencers como líderes de opinión, por lo que muchas empresas los contratan y la comunidad a la que se dirige lo escucha como prescriptores (Elorriaga y Monge, 2018).

Los consumidores no confían en la marca; ellos confían en las recomendaciones que les proporcionan otros consumidores que ya han experimentado dicho producto o marca. Importa mucho el nivel de satisfacción que un cliente tiene luego de probar el producto, haciendo así que el Boca-Boca sea el mejor método publicitario que pueda aplicarse.

\section{Marco Teórico}

El marco teórico estará conformado por 3 partes fundamentales; en la primera parte se hablará sobre las redes sociales, definición, funciones, principales características y utilidad; la segunda parte será sobre los influencers, personalidad, marca y suscriptores, todo esto con la finalidad de proporcionar mayores conocimientos acerca de este tema tan interesante; para finalizar la tercera parte tratará sobre los influencers ecuatorianos y se detalla el tipo de contenido en el que se desenvuelven.

\section{Redes Sociales}

Las definiremos como la unión de distintos puntos (usuarios) en distintos lugares con distintos gustos y prioridades pero que los unen una misma cosa; socializar sus intereses y hacerse conocer independiente de su naturaleza, raza o creencia, lo que importa es la experiencia que se desea compartir.

En función a esto, en general se diría que: la utilización de las redes y la diversidad de herramientas existentes forman un canal comunicacional el cual permite poner en contacto a millones de personas cada día y con mucha rapidez (Casaló, Flavián y Guinalíu, 2012).

En medio del importante auge de las redes sociales y su forma estructural, una gran cantidad de organizaciones han lanzado sus propias páginas en las distintas plataformas según se encuentra su segmento identificado, desarrollan sus perfiles en función de lo que pueda ser más atractivo para los usuarios y los nichos, para lo cual crean contenido en el que se pueda interactuar y compartir las experiencias que representen un ícono/referente entre los consumidores que esperan sus publicaciones, aplicando así algún tipo de valor añadido, obteniendo los beneficios que provienen de usar dichas redes. 
Es muy común ver como en la actualidad son más los profesionales y académicos que están preocupados por analizar las Redes Sociales, gestionarlas y organizarlas para poder promocionar marcas de distintas organizaciones.

Para Okazaki, Rubio y Campo (2012) son muy pocos los profesionales e investigadores que se preocupan por analizar a fondo este fenómeno, en las pocas investigaciones que se han realizado sobre este tema se puede determinar que se encuentran fundamentadas a través de aspectos cualitativos principalmente en la naturaleza de estas y en las relaciones con la red.

Es importante destacar que en una de las investigaciones pioneras realizadas por (Moreno, Jelenchick, Koff y Eickhoff, 2012) sobre las motivaciones de los universitarios por acceder a las redes sociales, registrarse y participar en ellas se descubrió que es la confianza que tienen al internet y la necesidad al sentirse parte de un grupo son algunos de los factores que los motivan y los llenan de actitud para poder ser parte de ellas.

García, Hoyo y Fernández (2014) desarrollaron dos investigaciones, en la primera evaluaron aspectos como la satisfacción y el uso de las redes sociales en jóvenes y los resultados que se obtuvieron indicaron que la gran parte de los encuestados pasan varias horas conectados, el objetivo de ellos es hacer nuevos amigos o reencontrarse con amigos del pasado.

Posterior a esto en la segunda investigación se quería determinar la satisfacción y el uso de los estudiantes de las redes sociales; los resultados aquí fueron de que se encontraron diferencias significativas entre sexos, puesto que la mayoría de los jóvenes comparte diferente tipo de información y experimentan inconformidad al saber que las empresas pueden obtener su información personal, ante esto se plantea la posibilidad de que los jóvenes puedan proteger su información a través de perfiles privados, de esta manera las empresas no podrían obtener información de los usuarios y ellos obtendrían satisfacción nuevamente al usar dichas redes de manera más segura.

Un aspecto importante a considerar es la teoría de los seis grados descrita por Malaver, Rivera y Álvarez (2010) en el artículo científico "La ciencia, la conectividad y la sociedad"; en él, los autores hacen referencia a la teoría de Duncan Watts, en la que se indica que cada persona tiene más o menos 100 amigos en su lista de contactos en redes sociales, a lo largo de la vida estos contactos pueden ir aumentando o reduciendo, pudiendo llegar a tener alrededor de $100 \mathrm{o}$ 200 personas, considerablemente si estas 100 personas nos presentan a sus 100 contactos (amigos) la lista incrementa y se llegaría a tener 10.000 amigos en la nueva lista, este proceso se lo desarrolla hasta llegar al sexto nivel en el cual se llegaría a tener 1 billón de contactos, con esto se resalta la posibilidad de que cada persona puede conocer a otra persona de diferente parte del mundo mediante la red social y su lista de contactos, es este el motivo por el cual se denomina a esta teoría de los seis grados.

Las redes sociales son muy útiles; es por esta razón que para su mayor beneficio y utilización se clasifican de la siguiente manera:

- Redes sociales profesionales: ayudan a las Pymes a ampliar su lista de contactos en el ámbito empresarial y a la búsqueda de oportunidades e interacción entre las personas. 
- Redes sociales generalistas: son aquellas personas que crean perfiles con la finalidad de realizar amigos y ponerse en contacto con ellos.

- Redes sociales especializadas: en ellas se comparten actividades económicas, deportivas, sociales para satisfacer la necesidad de personas con características similares. 
A continuación, se dará una descripción de las redes sociales más utilizadas las mismas que serán tomadas como referencia para realizar la metodología de la presente investigación:

Tabla 1

\section{Redes Sociales}

\section{Facebook}

\section{Instagram}

Es la red social más exitosa y Su principal orientación es el utilizarla presenta las siguientes características:

*Facilidad para que los usuarios puedan compartir fotos, videos $\mathrm{o}$ links.

*Su interfaz es sencilla facilita el acceso a la red a un usuario con poca experiencia.

* La experiencia en los mensajes es única, no necesita de terceros para realizar una comunicación en tiempo real.

*Entrega facilidad para que las empresas, los personajes públicos o las personas emprendedoras puedan ganar dinero a través de esta red social (Phillip y Fiona, 2017). desde dispositivos móviles, además se una versión únicamente de lectura. Sus aracterísticas son las siguientes:

* Se enfoca en permitir a las personas subir fotos y retocarlas con los filtros fotográficos que tiene.

*Los usuarios de esta red social se aman seguidores.

* Permite a los usuarios estar pendiente de las personas que se deseen ya sea por admiración o gusto.

* Permite usar hashtags, para que personas con gustos similares puedan encontrarse muy fácilmente (Remache,

Lascano, y Medina, 2018).

Twitter
Presenta las siguientes
características:
*La comunicación es de forma
bidireccional es decir el usuario
elige con quien desea
relacionarse.
*Sus mensajes están limitados
en un total de 140 caracteres.
*A diferencia de Facebook a
los usuarios de Twitter se los
denomina seguidores
"followers"
"followed".
*Twitter es muy divertido, pero
todo depende el uso y del valor
que cada persona le otorgue a
esta red social.
*Hace que las personas que la
usan se sientan mucho más
cerca de los seguidores de los
que se encuentran separados
por la distancia.
*Esta red social es más
eficiente, es decir la
información llega de forma más
rápida y de calidad.
avany Zhapann

\section{YouTube}

YouTube es una de las redes sociales más multitudinaria para poder compartir videos. Sus características son las siguientes:

*Es considerada como la red social más exitosa, en ella los aficionados pueden subir y compartir de manera rápida videos que en cuestión de segundos se pueden hacer virales alcanzando miles de reproducciones. * Se busca alcanzar el mayor número de suscriptores para poder obtener beneficios económicos.

*Los que comparten sus videos en YouTube y se hacen conocidos llevan la denominación de YouTubers.

Elaboración: Geovanny Zhapán 


\section{Influencer(s)}

Son aquellas personas que logran influir en las decisiones de su audiencia gracias al posicionamiento de su marca personal obtenido a través de sus redes sociales hacen el papel de prescriptores de la marca a la cual representa al ser contratado. Para lograr un acercamiento a los consumidores es necesario saber que el consumidor no compra el producto sino la experiencia con ese producto; es decir, la experiencia que otra persona (influencer) tiene con el producto acelera la decisión de compra (Gómez B. , 2018).

Tratan de persuadir a los consumidores indicando las características, beneficios y resultados del producto. Su trabajo es mostrar el elemento diferenciador de la marca en relación con la competencia de manera sutil y/o imperceptible hasta llegar al punto de que una comunidad entera pueda tener un sentido de pertenencia por la marca (Fernández, Hernández y Sanz, 2018).

La personalidad de cada influencer sería imposible describir uno por uno ya que existen en gran cantidad en el país. Pero lo más importante es que el usuario se sienta identificado con el prescriptor de cualquier marca a la que represente. No hay un patrón o regla que nos indique una personalidad que conceptualice a esas personas.

Se tratará de explorar, generalizar y ejemplificar buscando así un modo para abordar el análisis que al notarse de una mejor forma resulta simple en exceso (Piedra y Meana, 2017). Por otra parte, si se pueden clasificar y encajar a cada prescriptor según el contenido, ya sea contenido deportivo, salud, fitness, entretenimiento, informativo, gamers.

La marca de un prescriptor es: él mismo como persona; la imagen y el contenido que ofrece es la base que construye su marca, éstas la mantienen y la posicionan en el mercado que vale decir; se encuentra saturado pero que no deja de presentar una oportunidad para nuevos prescriptores.

El posicionarse determinará el tiempo que durará en esta labor puesto que hay influencers que fueron etéreos y otros que, aunque ya se retiraron de la vida pública aún son referentes en las redes sociales, otros que aún permanecen y quienes apenas surgen y tratan de ganar un espacio en el mercado.

La visión es crear la lealtad de una comunidad y luego vender los servicios a una empresa que esté proyectada a aumentar sus ventas pero que su marca ya sea reconocida para potenciarse aún más o por lo menos que sea confiable. Se muestra las características y beneficios del producto y se repite el proceso (aplica-enjuaga-repetir) (Schlesinger, Cervera y Calderón, 2014).

Los prescriptores son aquellas personas que han logrado mediante su carisma y dedicación influenciar en la decisión de compra en una comunidad, ganar adeptos, alcanzar credibilidad, lealtad de sus seguidores, intimar con las personas mediante una pantalla colgados de la red (Sanz, Ruíz y Pérez, 2013). Los prescriptores generan ingresos mediante su recomendación de usar tal o cual servicio de la empresa que lo contrató. En Ecuador se conocía mucho el asunto del canje (servicios o productos a cambio de publicidad "gratuita") en la 
actualidad ya no se está dando esto debido a que mayor es el beneficio de la empresa que del influencer contratado.

\section{Influencers ecuatorianos}

En la actualidad se ha podido observar cómo ha evolucionado el concepto de publicidad mediante el uso de influencers; que, por su alta aceptación del público han logrado que muchas de las marcas a las que representan queden posicionados en el mercado (Ortiz y Suing, 2016). Las empresas ecuatorianas están aprovechando este nuevo medio de publicidad en sus productos, pero es importante destacar que a la hora de elegir a un influencer o de elaborar una campaña, se deben seguir ciertas recomendaciones:

1. Segmentar el público objetivo: no es necesario que sean clientes a los que se dirija esta estrategia, puede ser dirigido a un mercado potencial. Al hacer una correcta segmentación hará que la potencial compra pueda darse mostrando un contenido que de valor y evitando así que el mensaje se vuelva molesto para ellos nos menciona (Xu y Coatney, 2015).

2. Identifica al influencer que necesitas: contratar al prescriptor con gran número de seguidores es un gran error que cometen distintas marcas; no es solamente el número de seguidores lo que se debe tomar en consideración. Es el público al que pertenece el prescriptor el valor agregado lo que se tomará en cuenta porque está especializado en ese sector (Djafarova y Rushworth, 2017).

3. Creatividad: en este punto la empresa debe ser creativa al momento de seleccionar el mensaje publicitario y el tipo de publicidad que quiere transmitir al público, debe dejar que el influencer se desenvuelva de manera natural para que todo fluya y no parezca sacado de un libreto, de esta forma el prescriptor puede compartir una historia o experiencia con el producto (Freberg, Grahamb, McGaughey y Freberg, 2011).

4. Micro-Influencer: este tipo de prescriptor es el que tiene una influencia especializada en un nicho, por lo que contactar con ellos puede aumentar la efectividad del plan de marketing digital. Cuanto menos tiene, más efectivo en lo que se planea; menos, es más, más especializado y menos errores en qué preocuparse puesto que hay mejor comunicación (Pérez y Clavijo, 2017)

\section{Contenido de los influencers}

En redes sociales se ha podido determinar que los influencers se destacan por dirigirse hacia un tipo de contenido específico, siendo ese su punto fuerte para poder ganar mayor cantidad de seguidores, es importante señalar que en otros países los influencers desarrollan una gran cantidad de contenido para alimentar sus redes sociales, páginas web o blogs, pero este no es caso de Ecuador puesto que el contenido que genera la mayoría es de entretenimiento (Castelló y Pino, 2015). A continuación, se detallarán diversos contenidos de los influencers:

$\checkmark$ Moda y belleza: las influencers dan a conocer tips de belleza, muestran cómo realizarse algún maquillaje, cual es el outfit adecuado para una salida especial, es decir, proporcionan contenido para chicas, el mismo que es de mucho beneficio y que les permite que su audiencia quede satisfecha con la información que les están transmitiendo. 
$\checkmark$ Salud/fitness: el material de este tipo de contenido es netamente saludable, es decir, muestran videos cortos sobre rutinas de ejercicios diarios que se pueden realizar para mantenerse en forma, además proporcionan recetas saludables para ayudar a que sus seguidores se incentiven y se animen llevar una vida más saludable.

$\checkmark$ Informativo: este contenido por lo general es utilizado en blogs en los cuales se comparte diverso tipo de información relevante para cada segmento hacia el cual se ha planteado llegar el influencer.

$\checkmark$ Entretenimiento: se lo realiza a través de videos, en los cuales se cuentan historias graciosas, o se los realiza buscando temáticas actuales y de tendencia para poder llegar más rápido a su audiencia, estos videos son subidos en sus redes sociales y algunos influencers cuentan con canales de YouTube en donde también los comparten.

$\checkmark$ Deportivo: este tipo de contenido es muy común para aquellos influencers que son deportistas, puesto que aquí ellos comparten mucha información sobre los productos que usan, o inclusive dan recomendaciones e incentivan a las personas a practicar algún tipo de deporte.

\section{Metodología}

Se usa la metodología de investigación de mercados cualitativa con el fin de visualizar las reacciones y necesidades del consumidor. Esto representa una utilidad para el diseño de nuevos productos y su evaluación en los procesos productivos, el entender algunas características de la producción y el determinar la pertinencia de las alternativas de comercialización (Alonso, y otros, 2017)

El método utilizado en la investigación es de tipo cualitativo, se aplicará como técnica una guía de observación online, en la que se analizaran indicadores importantes para determinar si el comportamiento de los influencers es similar y los motivos por los que las marcas han adoptado esta nueva manera de realizar publicidad.

Los indicadores a evaluar son los siguientes:

$>$ Número de seguidores / suscriptores en redes sociales: en este indicador se podrá determinar la aceptación que tienen los influencers puesto que el número de seguidores es un factor muy importante para las empresas al momento de elegir al prescriptor que promocione sus productos o servicios.

$>$ Interacciones positivas o negativas: es importante tener conocimiento de las interacciones que tienen los influencers con sus seguidores, de esto depende el éxito de la publicidad.

$>$ Mensaje publicitario: se conocerá el tipo de mensaje que emita cada prescriptor, es decir, algunos se basan en experiencias propias mientras que otros se regulan a las disposiciones de la marca.

$>$ Marcas publicitarias: en este indicador se podrá conocer cuáles son las marcas que han trabajado con los influencers.

$>$ Calidad de las fotos o videos: es fundamental tener presente este indicador, porque a mayor calidad en su contenido mejor será la interacción con sus seguidores.

$>$ Forma en la que realizan la publicidad: grado de emoción o naturalidad que tenga el prescriptor al realizar la publicidad de las marcas. 


\section{Clasificación de los influencers ecuatorianos}

Para una mayor comprensión de estos prescriptores y su patrón conductual; se ha visto necesario la elaboración de una clasificación donde también pueda ser más didáctico su estudio. La clasificación se hará en función del contenido que presentan los sujetos de estudio en sus vídeos y posterior a esto se realizará un cuadro recabando los datos más relevantes que se han obtenido de dichos influencers.

A continuación, se detalla a los influencers ecuatorianos que se tomarán como referencia para desarrollar la metodología de la investigación, a los cuales se clasificará según el contenido:

- Efraín Ruales: Entretenimiento

Se ha caracterizado por utilizar este tipo de contenido al caracterizar a varios personajes logrando que sus videos que sean graciosos y entretenidos.

- Emma Guerrero: Entretenimiento Decidió optar por el entretenimiento y fue este tipo de contenido el que le permitió obtener mayor reconocimiento y posicionar su marca.

- Anthony Swag: Entretenimiento

De la misma forma que los influencers anteriores decidió crear contenido gracioso que le ayudado a ganar fama y a incrementar el número de seguidores.

- Michela Pincay: Moda y belleza Su principal potencial es el crear contenido sobre moda, belleza, estilos de vida, personalidad, entre otros, con ellos ayuda a muchas de sus seguidoras a saber que elegir.

- Felipe Crespo: Entretenimiento Apostó por la realización de contenido de entretenimiento, sus videos son muy particulares, porque él va a discotecas a realizar preguntas al público, es decir, interactúa mucho con los seguidores.

- María Teresa Guerrero: Deporte

Esta Influencer decidió llegar al mercado a través del deporte como contenido, ahí ella enseña y motiva a sus seguidores a practicar algún deporte.

- Erika Vélez: Moda y belleza Enseña trucos de maquillaje, moda y belleza, gracias a este contenido ha logrado que satisfacer a muchas de sus seguidoras.

- Nancy Risol: Entretenimiento

Pese a que es nueva en la incursión del trabajo en redes sociales, esta carismática chica decidió realizar contenido de entretenimiento, el mismo que le ha permitido crecer significativamente el número de seguidores. 


\section{Desarrollo de la metodología}

Se realizó la guía de observación online para determinar patrones de comportamientos de los influencers y de las marcas:

Tabla 2

Datos destacados obtenidos en la metodología.

\begin{tabular}{|c|c|c|c|c|c|}
\hline $\begin{array}{l}\text { Indicadores } \\
\text { Influencers }\end{array}$ & $\begin{array}{l}\text { Seguidores / } \\
\text { suscriptores }\end{array}$ & $\begin{array}{l}\text { Contenido no } \\
\text { comercial }\end{array}$ & $\begin{array}{l}\text { Marcas con las } \\
\text { que ha trabajado }\end{array}$ & $\begin{array}{l}\text { Plataforma } \\
\text { publicitaria }\end{array}$ & Forma de la publicidad \\
\hline $\begin{array}{l}\text { Efraín } \\
\text { Ruales }\end{array}$ & $\begin{array}{l}\text { Facebook: } \\
\text { 136.032 } \\
\text { seguidores } \\
\text { Instagram: } \\
1^{\prime} 474.177 \\
\text { seguidores } \\
\text { YouTube: } \\
43.628 \\
\text { suscriptores } \\
\text { Twitter: } \\
491.791 \\
\text { seguidores }\end{array}$ & Entretenimiento & $\begin{array}{l}\text { - On moda boutique } \\
\text { - Oriental: fideos } \\
\text { rapiditos } \\
\text { - Glovo } \\
\text { - Mi juguetería } \\
\text { - Pilsener } \\
\text { - Phraa } \\
\text { - Garnier fructis } \\
\text { - El oro mega } \\
\text { diverso } \\
\text { - Aeropuerto de } \\
\text { Guayaquil } \\
\text { - Claro } \\
\text { - Adidas } \\
\text { - Head \& Shoulders }\end{array}$ & $\begin{array}{l}\text { Instagram y } \\
\text { Twitter }\end{array}$ & $\begin{array}{l}\text { La publicidad la realiza mediante fotos y videos en los cuales } \\
\text { comparte experiencias e información proporcionada por la } \\
\text { empresa que solicita sus servicios. }\end{array}$ \\
\hline $\begin{array}{l}\text { Emma } \\
\text { Guerrero }\end{array}$ & $\begin{array}{l}\text { Facebook: } \\
37.000 \\
\text { seguidores } \\
\text { Instagram: } \\
386.101 \\
\text { seguidores } \\
\text { Twitter: } 430 \\
\text { seguidores }\end{array}$ & Entretenimiento & $\begin{array}{l}\text { - Head \& Shoulders } \\
\text { - Ih Ecuador } \\
\text { (Aprendizaje de } \\
\text { ingles) } \\
\text { - ATM Guayaquil } \\
\text { - Frutaris } \\
\text { - Moda Koaj } \\
\text { - Dr. Hernán Pérez } \\
\text { - Flormar Ecuador } \\
\text { - Sony }\end{array}$ & Instagram & $\begin{array}{l}\text { Emma Guerrero realiza la publicidad con totos y videos, lo que } \\
\text { la diferencia a ella es que el contenido es de experiencias que ha } \\
\text { vivido, logrando así que los seguidores se muestren interesados } \\
\text { en adquirir los productos que promociona. }\end{array}$ \\
\hline $\begin{array}{l}\text { Anthony } \\
\text { Swag }\end{array}$ & $\begin{array}{l}\text { Facebook: } \\
\text { 2'504.289 } \\
\text { seguidores } \\
\text { Instagram: }\end{array}$ & Entretenimiento & $\begin{array}{l}\text { - Salaberry } \\
\text { Restaurant } \\
\text { - Condor Oainting } \\
\text { LLC }\end{array}$ & Instagram & $\begin{array}{l}\text { El contenido en su totalidad parte de la empresa, es decir, el } \\
\text { influencer no se base en experiencias obtenidas debido a que en } \\
\text { sus redes sociales trata de posicionarse como él como marca. }\end{array}$ \\
\hline
\end{tabular}




$\begin{array}{ll}75.963 & \text { - Naturama Ecuador } \\ \text { seguidores } & \text { - Labahia.ec } \\ \text { YouTube: } & \text { - Kriscar Taxi } \\ 732.527 & \text { - Tiendas El Garaje } \\ \text { suscriptores } & \text { - Stikers_ec }\end{array}$

(t)

Twitter:

15.010

seguidores

Facebook:

\begin{tabular}{|c|c|c|c|c|}
\hline & Facebook: & & - Creandoeventos & \\
\hline Michela & 57.862 & & - Derma Nutrix & \\
\hline \multirow[t]{13}{*}{ Pincay } & seguidores & Moda y belleza & Ecuador & \\
\hline & Instagram: & & - Sedal & Instagram y \\
\hline & 11.382 & & - Íntima & Twitter \\
\hline & seguidores & & - Catalogo mi & \\
\hline & Twitter: & & Ángel & \\
\hline & 705.195 & & - Mind Agency & \\
\hline & seguidores & & - Teep Party & \\
\hline & & & - Hola Tv & \\
\hline & & & - Nueva mujer & \\
\hline & & & - Cruz Azul & \\
\hline & & & - Kotex & \\
\hline & Facebook: & & - MentoringProject & \\
\hline & 274.695 & & - Lexa & \\
\hline \multirow{9}{*}{$\begin{array}{l}\text { Felipe } \\
\text { Crespo }\end{array}$} & seguidores & & - Samsunglatin & \\
\hline & Instagram: & Entretenimiento & - Prouniversitarios & Instagram \\
\hline & 176.356 & & - Restaurante & \\
\hline & seguidores & & Corvel & \\
\hline & YouTube: & & -The Camp Raven & \\
\hline & 673.492 & & Ecuador & \\
\hline & suscriptores & & & \\
\hline & Twitter: 2.243 & & & \\
\hline & seguidores & & & \\
\hline \multirow{5}{*}{$\begin{array}{l}\text { María } \\
\text { Teresa } \\
\text { Guerrero }\end{array}$} & & & - Colgate & \\
\hline & Instagram: & & - Ironman Triathlon & \\
\hline & 1'177.116 & Deporte & - Roka Sports & \\
\hline & seguidores & & - Safettiecuador & Instagram y \\
\hline & Twitter: & & - Nutribela & Twitter \\
\hline
\end{tabular}

Con la mayoría de las marcas ha trabajado a través de fotografías en las cuales expresa confianza y seguridad en el producto que menciona y recomienda. También ha trabajado a través de videos con las marcas Sedal e Íntimas, esto si como comerciales que han salido en televisión nacional y ha compartido en su red social Instagram para captar nuevos mercados.

En la publicidad este influencer comparte información proporcionada por la empresa, es decir su publicidad no se basa en experiencias obtenidas por la marca. 


\begin{tabular}{|c|c|c|c|c|c|}
\hline & $\begin{array}{l}1 ’ 430.788 \\
\text { seguidores }\end{array}$ & & $\begin{array}{l}\text { - Hotel del Parque } \\
\text { - Felt Bicycles } \\
\text { - BioSil } \\
\text { - Feder Mut } \\
\text { - Equivida } \\
\text { - Toscana Pastas } \\
\text { - Nike women }\end{array}$ & & \\
\hline Erika Vélez & $\begin{array}{l}\text { Facebook: } \\
25.616 \\
\text { seguidores } \\
\text { Instagram: } \\
\text { 1'332.052 } \\
\text { seguidores } \\
\text { Twitter: } \\
948.304 \\
\text { seguidores }\end{array}$ & (1) & $\begin{array}{l}\text { - Cosas Ecuador } \\
\text { - Medical Medium } \\
\text { - Milani Ecuador } \\
\text { - De prati } \\
\text { - Topcloset } \\
\text { - Biosil } \\
\text { - Saviloe }\end{array}$ & $\begin{array}{l}\text { Instagram y } \\
\text { Twitter }\end{array}$ & $\begin{array}{l}\text { Sus mensajes publicitarios se basan más en la experiencia que } \\
\text { ella ha tenido con la marca, transmitiendo seguridad al público al } \\
\text { indicar que si a ella le funcionó a sus seguidores también les } \\
\text { funcionara. }\end{array}$ \\
\hline Nancy Risol & $\begin{array}{l}\text { Facebook: } \\
\text { 1'982.854 } \\
\text { seguidores } \\
\text { Instagram: } \\
303.191 \\
\text { seguidores } \\
\text { YouTube: } \\
\text { 1'184.426 } \\
\text { suscriptores } \\
\text { Twitter: } 341 \\
\text { seguidores }\end{array}$ & Entretenimiento & - Grow Hair & Instagram & $\begin{array}{l}\text { La publicidad que realizó se basó en el contenido que la empresa } \\
\text { le proporcionó, la marca con la que trabajó no es muy } \\
\text { reconocida, esto es porque ella recién está empezando a } \\
\text { incursionar como influencer. }\end{array}$ \\
\hline
\end{tabular}

Fuente: Plataformas Instagram, Facebook, Twitter, YouTube

Elaboración: Geovanny Zhapán 


\section{Análisis de resultados}

Se encontró como resultado: que, en su mayoría, los influencers ecuatorianos se dedican al entretenimiento, apuestan a aquello para poder ganar más seguidores en cualquiera de sus plataformas, mientras que en un porcentaje menor se dedican a la información deportiva, moda, salud, fitness.

\section{Efraín Ruales:}

De acuerdo a las publicidades que ha realizado se puede decir que Efraín Ruales realiza los contenidos publicitarios en un 50\% con la información y el dialogo de la empresa (marca) y 50\% bajo las experiencias que ha obtenido al utilizarla.

\section{Emma Guerrero:}

El $100 \%$ del contenido publicitario de esta influencer es en base a sus experiencias y resultados, puesto que demuestra que el producto o servicio es de calidad.

\section{Anthony Swag:}

Su contenido publicitario se basa $100 \%$ a dialogo que le entrega la marca, es decir, no se basa en experiencias vividas con el producto.

\section{Michela Pincay:}

Esta influencer realiza las publicidades un $75 \%$ bajo a las experiencias o percepciones que ha tenido de la marca y en un $25 \%$ bajo diálogo o contenido que le proporciona la empresa.

\section{Felipe Crespo:}

La publicidad la realiza bajo las directrices de la marca, esto se puede notar en la descripción de las imágenes publicitarias que sube en las redes sociales.

\section{María Teresa Guerrero:}

La publicidad la realiza al 100\% con información proporcionada por la marca, es decir es la misma marca su patrocinador en los eventos deportivos.

\section{Erika Vélez:}

El contenido publicitario de esta influencer se basa $50 \%$ en información proporcionada por la empresa y $50 \%$ de las experiencias que ha tenido con la marca.

\section{Nancy Risol:}

Ha realizado únicamente 1 publicidad de la cual la información es al 100\% proporcionada por la marca.

\section{Conclusiones}

Se demostró que en Ecuador existe un crecimiento de influencers exponencial, con contenido no comercial de entretenimiento para poder captar más seguidores como un medio que ayude a alcanzar la meta fija; que las marcas decidan pautar con ellos en sus 
espacios sociales. Este incremento se encontró en toda la clasificación señalada para ser objeto de estudio.

Mediante esto se puede concluir qué, los prescriptores tienen un patrón de comportamiento que los hace referentes al momento de buscar distracción (entretenimiento) por parte del consumidor y es con lo que han logrado posicionar su marca personal, gracias al contenido no comercial que cuelgan en sus plataformas sociales: entretenimiento, belleza y deporte.

Limitaciones: no se encontró información de todos los influencers ecuatorianos. Es imposible poder colocar a todos los influencers por lo que se ha tomado referentes de los mismos.

Queda abierta la oportunidad en investigaciones futuras encontrar el motivo por el cual los influencers ecuatorianos según los resultados encontrados en la observación; no son tomados como un referente informativo (prescriptores) en su gran mayoría, sino que, estos se toman para el simple entretenimiento, lo cual podría deberse a instancias culturales.

\section{Bibliografía}

Alonso, J., Arboleda, A., Rivera, A., Mora, D., Tarazona, R., \& Ordoñez, P. (2017). Técnicas de investigación cualitativa de mercados aplicadas al consumidor de fruta en fresco. Estudios Gerenciales, 412-420. doi:https://doi.org/10.1016/j.estger.2017.10.003

Arce, M., \& Cebollada, J. (2013). Elección de canal de compra y estrategia multicanal: internet vs. tradicional. Aplicación a la compra en una cadena de supermercados. Cuardernos de Economía y Dirección de la Empresa, 108-122.

Casaló, L., Flavián, C., \& Guinalíu, M. (2012). Redes sociales virtuales desarrolladas por organizaciones empresariales: antecedentes de la intención de participación del consumidor. Cuadernos de Economía y Dirección de la Empresa, 42-51.

Castelló, A., \& Pino, C. d. (2015). La comunicación publicitaria con influencers. Revista Digital de Marketing Aplicado, 21-50. doi:https://doi.org/10.17979/redma.2015.01.014.4880

Djafarova, E., \& Rushworth, C. (2017). Exploring the credibility of online celebrities' Instagram profiles in influencing the purchase decisions of young female users. Computers in Human Behavior, 1-7.

Duque, E., \& Ramírez, P. (2014). Evolución conceptual y relación entre involucramiento y lealtad. Suma de Negocios, 169-179.

Elorriaga, A., \& Monge, S. (2018). La profesionalización de los youtubers: el caso de Verdeliss y las marcas. Revista Latina de Comunicación Social, 37-54. doi:10.4185/RLCS-2018-1244

Fernández, J., Hernández, V., \& Sanz, P. (2018). Influencers, marca personal e ideología política en Twitter. Cuadernos.Info, 19-37. doi:https://doi.org/10.7764/cdi.42.1348

Freberg, K., Grahamb, K., McGaughey, K., \& Freberg, L. (2011). Who are the social media influencers? A study of public perceptions of personality. Public Relations Review, 90-92. 
García, M., Hoyo, M. D., \& Fernández, C. (2014). Engaged youth in the internet. The role of social networks in social active participation. Comunicar, 35-43. doi:https://doi.org/10.3916/C43-2014-03

Gómez, B. (2018). El influencer: herramienta clave en el contexto digital de la publicidad engañosa. Revista de Ciencias Sociales, 149-156. doi:http://dx.doi.org/10.17502/m.rcs.v6i1.212

Malaver, M., Rivera, H., \& Álvarez, L. (2010). La ciencia de las redes, la conectividad y la sociedad. Semestre Económico, XIII(26), 149-157.

Moreno, B., \& Calderón, M. (2017). Comportamiento del consumidor en la co-creación de valor y su relación con la satisfacción en el entorno universitario: una aplicación a la Universidad de Ibagué (Colombia). Revista Facultad de Ciencias, 204-206.

Moreno, M., Jelenchick, L., Koff, R., \& Eickhoff, J. (2012). Depression and Internet Use among Older Adolescents: An Experience Sampling Approach. Psychology, III, 743-748. doi:http://dx.doi.org/10.4236/psych.2012.329112

Okasaki, S., Rubio, N., \& Campo, S. (2012). Antecedentes de las Redes Sociales como canales de comunicación promocional para los jóvenes. Revista Española de Investigación de Marketing ESIC, 31-48.

Ortiz, C., \& Suing, A. (2016). La televisión ecuatoriana: pasado y presente. Razón y Palabra, 135-152.

Pérez, C., \& Clavijo, L. (2017). Comunicación y social media en las empresas de moda ASOS como caso de estudio. Prisma Social Revista de Ciencias Sociales, 226-258.

Phillip, O., \& Fiona, J. (2017). Materialists on Facebook: the self-regulatory role of social comparisons and the objectification of Facebook friends. Heliyon, 1-24. doi:https://doi.org/10.1016/j.heliyon.2017.e00449

Piedra, E. d., \& Meana, R. (2017). Redes sociales y «Fenómeno Influencer». Reflexiones desde una perspectiva psicológica. Revista de Ciencias Humanas y Sociales, 443469.

Remache, A., Lascano, M., \& Medina, R. (2018). Estrategia de marketing basada en el uso de Instagram para la publicidad de ropa interior en Tungurahua, Ecuador. Revista Iberoamericana de Contaduría, Economía y Administración, 1-28.

Salas, M. I. (2009). La publicidad en redes sociales: de lo invasivo a lo consentido. ICONO14, 75-84.

Sanz, S., Ruíz, C., \& Pérez, I. (2013). Factores determinantes de la lealtad al proveedor de servicios turísticos online. Contaduría y Administración, 279-302.

Schlesinger, Cervera, \& Calderón. (2014). El papel de la confianza, la imagen y los valores compartidos en la creación de valor y lealtad: aplicación a la relación egresadouniversidad. Revista Española de Investigación de Marketing ESIC, 126-139. doi:http://dx.doi.org/10.1016/j.reimke.2014.06.001

Taramona, R. (2018). Influencers digitales: disrupción de la fama, la publicidad y el entretenimiento en las redes sociales. Revista de Estudios de Juventud, 75-92.

Villena, E. (2018). El influencer de moda como sujeto de relaciones públicas: recursos y herramientas. Redmarka Revista de Marketing Aplicado, I(022), 115-128. doi:https://doi.org/10.17979/redma.2018.01.022.4938

$\mathrm{Xu}, \mathrm{X}$., \& Coatney, K. (2015). Product market segmentation and output collusion within substitute products. Journal of Economics and Business, 1-15. 